Copyright

by

Razieh Nokhbeh Zaeem

2010 
The Thesis committee for Razieh Nokhbeh Zaeem certifies that this is the approved version of the following thesis

\title{
Contract-based Data Structure Repair Using Alloy
}

\author{
APPROVED BY \\ SUPERVISING COMMITTEE:
}

Sarfraz Khurshid, Supervisor

Kathryn S. McKinley 


\title{
Contract-based Data Structure Repair Using Alloy
}

\author{
by \\ Razieh Nokhbeh Zaeem, B.S.
}

\author{
THESIS \\ Presented to the Faculty of the Graduate School of \\ The University of Texas at Austin \\ in Partial Fulfillment \\ of the Requirements \\ for the Degree of \\ MASTER OF SCIENCE IN ENGINEERING
}

THE UNIVERSITY OF TEXAS AT AUSTIN

May 2010 
To my parents, Fatemeh and Davoud, and to my husband, Mahdi, for their infinite love and support. 


\section{Acknowledgments}

This material is based upon work partially supported by the NSF under

Grant Nos. IIS-0438967, and CCF-0845628, and AFOSR grant FA9550-09-10351. 


\title{
Contract-based Data Structure Repair Using Alloy
}

\author{
Razieh Nokhbeh Zaeem, M.S.E. \\ The University of Texas at Austin, 2010
}

Supervisor: Sarfraz Khurshid

Contracts and specifications have long been used in object-oriented design, programming and testing to enhance reliability before software deployment. However, the use of specifications in deployed software is commonly limited to runtime checking where assertions form a basis for detecting incorrect program states to terminate the erroneous executions. We present a contract-based approach for data structure repair, which repairs erroneous states in deployed software. The key novelty is the support for rich behavioral specifications, such as those that relate pre-states with post-states of a method to accurately specify expected behavior and precise repair. The approach is based on the view of a specification as a non-deterministic implementation. The key insight is to use any correct state mutations by an otherwise erroneous execution to prune non-determinism in the specification, thereby transmuting the specification to an implementation that does not incur a prohibitively high performance penalty. While invariants, pre-conditions 
and post-conditions could be provided in different modeling languages, we leverage the Alloy tool-set, specifically the Alloy language and the Alloy Analyzer for systematically repairing erroneous states. Four different algorithms are presented and implemented in our data structure repair framework. These algorithms can repair a medium sized erroneous data structure in a few seconds. We introduce repair guide annotations defined by the user to improve the accuracy and performance of the repair mechanism. Experiments using complex specifications show the approach holds much promise in increasing software reliability. 


\section{Table of Contents}

Acknowledgments $\quad$ v

Abstract vi vi v

List of Tables ix

List of Figures $\quad$ x

Chapter 1. Introduction 1

1.1 Contributions ...................... 5

$\begin{array}{lll}\text { Chapter 2. Example } & 7\end{array}$

$\begin{array}{lll}\text { Chapter 3. Background } & 12\end{array}$

$\begin{array}{llr}\text { Chapter 4. Tarmeem } & 16\end{array}$

4.1 Definition of repair . . . . . . . . . . . . . . . . 16

4.2 Overview . . . . . . . . . . . . . . . . 17

4.3 Algorithms . . . . . . . . . . . . . . . . . . . 19

4.4 Instrumentation . . . . . . . . . . . . . . . . 32

$\begin{array}{lll}\text { Chapter 5. Evaluation } & 36\end{array}$

5.1 Singly Linked List . . . . . . . . . . . . . . . . . . . . . . . 36

5.2 ANTLR BaseTree . . . . . . . . . . . . . . . . . 41

Chapter 6. Conclusions and Future Work 45

$\begin{array}{ll}\text { Bibliography } & 48\end{array}$

$\begin{array}{ll}\text { Vita } & 54\end{array}$

viii 


\section{List of Tables}

4.1 Comparison of different repair Algorithms. . . . . . . . . . . . 32

5.1 Faults injected into the programs. Faults 1-7 apply to remove method of singly-linked list, and Faults 8-9 apply to deleteChild method of class BaseTree of ANTLR. . . . . . . . . . . . . .

5.2 Result of applying Tarmeem on different erroneous singly linked lists of size 10 and 20, and on corrupt BaseTree class of ANTLR package, maximum repair time allowed: 120 seconds. . . . . . 


\section{List of Figures}

2.1 Singly-linked list in Java. . . . . . . . . . . . . . . . . . . 8

2.2 An erroneous remove method for singly-linked list in Java. . . 9

2.3 Class invariant for LinkedList and post-condition for remove in Alloy. . . . . . . . . . . . . . . . . . . . 10

2.4 Linked list example. . . . . . . . . . . . . . . . . . . . . 11

2.5 Relational representation of the linked list example. . . . . . . 11

4.1 Tarmeem architecture. . . . . . . . . . . . . . . . . 18

4.2 Approach 1: the Basic method. . . . . . . . . . . . . . 21

4.3 (a) The erroneous output of remove (5) (b) The result of repairing the sample faulty output using Basic approach. . . . . . . 22

4.4 Approach 2: Iterative Relaxation. . . . . . . . . . . . . . . . . 23

4.5 (a) The erroneous output of remove (5) (b) The result of repairing the sample faulty output using Iterative Relaxation, Error Localization and Guided Error Localization. . . . . . . . . . . 24

4.6 Approaches 3 and 4: Error Localization. . . . . . . . . . . 26

4.7 Approaches 3 and 4: helper methods. . . . . . . . . . . . . . . 27

4.8 (a) Circular linked list: a hypothetical erroneous output of remove(5) (b) The result of repairing the faulty output using Guided Error Localization. . . . . . . . . . . . . . . . . . . 31

4.9 Linked List example pre- and post-state snapshots. . . . . . . 35

5.1 Class invariant for ANTLR BaseTree and post-condition for delete in Alloy. . . . . . . . . . . . . . . . . . . . . . . . . 42

5.2 Input grammar for ANTLR. . . . . . . . . . . . . . . . . 43

5.3 deleteChild method of class BaseTree. . . . . . . . . . . . . 43 


\section{Chapter 1}

\section{Introduction}

As software has become increasingly integrated with critical systems of high complexity, software failures have become prohibitively expensive and alarmingly frequent. There is now an urgent need for creating new methodologies that deliver reliable software at a lower cost. Much of the existing research effort is devoted to requirements, architecture, design, implementation and testing - activities that are performed before deployment of a software system. In contrast, little effort is devoted to developing methodologies that handle errors that arise during system executions after deployment. While requirements, architecture, design, implementation and testing are fundamental to develop correct programs, unfortunately not all faults are detected or fixed before deployment. The ability to handle runtime errors on-the-fly can bring a substantial increase in reliability.

The standard approach when an erroneous program state is detected at runtime, say due to an assertion violation, is to terminate the program, debug it if possible, and re-execute it. While this halt-on-error approach is useful to eliminate transient errors or for debugging purposes, it does not present a feasible solution for deployed software that is faulty and cannot be 
promptly debugged or re-deployed. A program that is functionally incorrect will continue to exhibit its failure(s) no matter how many times it is re-executed or on how many different machines it is executed. In such a situation, error recovery, and in particular repair of erroneous program state is necessary. In addition, errors in persistent data, such as a file system, also necessitate repair mechanisms $[2,3]$.

While repair mechanisms are not in standard use today, repair has featured in various systems over the last couple of decades $[2,3,13,27]$. However, a limitation of the traditional approaches is to use dedicated repair routines, which must be implemented for each system they are intended to work for. As a result, these routines are mostly ad-hoc and ill-understood.

Recent work introduced constraint-based repair, where data structure constraints are written using first-order logic $[9,10]$ or as Java assertions [11, $12,18]$ and are used as a basis for repairing erroneous states. While these approaches do not necessitate writing a dedicated repair routine, they have a basic limitation: data structure constraints are too weak for general error recovery. To illustrate, in object-oriented programs, the class invariant [23] (which defines the data structure constraints for the valid objects of the class) applies to the entry and exit points of all public methods - even though the precise behaviors of the methods may be very different. For example, consider an erroneous implementation of a method to insert an element into a binary tree-an acyclic data structure. Previous approaches $[10,18]$ to constraintbased repair would accept an empty tree as a valid structure since it satisfies 
the acyclicity constraint. However, an empty tree is unlikely to be a valid output of insert.

Constraint-based repair suffers from several limitations. First, all methods are restricted to use the same class invariants and hence the repair mechanism cannot reflect any specific property of the method. In inserting an element to the binary tree example mentioned earlier, the repair framework cannot detect or recover from states that the method fails to add the new element despite providing a valid binary tree as output. Second, the repair mechanism accepts any correct data structure (including the valid input data structure) as output of any method. Therefore, an empty method satisfies all needed properties according to repair mechanism.

Method contracts [26] naturally suit the repair process since they introduce class invariants, pre- and post-conditions and are widely used for other phases of software development. However, while they have long been used to improve software reliability in different phases of software development lifecycle (from accurately specifying requirements to design, implementation and testing), their common usage follows the same standard halt-on-error approach upon detecting an erroneous program state in a deployed software.

We present Tarmeem, a novel framework for contract-based data structure repair, which addresses the basic limitations of previous approaches to repair and is based on a novel role for rich behavioral specifications [21]: to repair erroneous executions. We use runtime specification violation as a basis of repairing the program state. Our insight is to transmute the contract 
specification - which permits a high degree of non-determinism - into an efficient implementation using the erroneous state. Any correct component of the erroneous state restricts the non-determinism in the specification and enables an efficient generation of a correct program state. While we support several specification languages, such as the Java Modeling Language [22], we translate the specifications to Alloy [15], transform the repair problem into a constraint solving problem and leverage the Alloy tool-set to provide systematic repair.

Tarmeem supports Alloy as the base modeling language. Alloy is a setbased specification language for describing structural properties. Alloy has a rich library and it is simple to define a large class of constraints in it, including set constraints, sequencing constraints, etc. Java Modeling Language (JML) is a behavioral interface specification language for Java modules. Support for JML through translation is made possible by the JForge [32] framework, which uses the Alloy back-end for scope-bounded checking of Java programs. Other languages similar to JML (such as Spec\# [5]) can be supported through translation as well.

Rich behavioral specifications accurately describe expected behavior and enable precise repair. Furthermore, it opens up a novel application of contracts in recovering programs from erroneous executions. We note that the use of contracts does not necessarily allow recovering from hardware errors, rather our focus is to recover from errors due to residual bugs or external events that mutate program state but not the execution environment or the contracts. 
While pre- and post-conditions have been used in repairing relational data and recovering from erroneous executions in databases [20,31], our work is distinguished in two aspects. Most importantly, previous work does not perform repair based on pre- and post-conditions but only aborts the transaction when expected relationship between pre- and post-states is not met. Secondly, our repair mechanism works on sophisticated pointer-based data structures which differs fundamentally from database schemas.

\subsection{Contributions}

We introduced the idea of contract-based repair in a one page extended abstract [34]. Our ECOOP2010 paper describes our approach in detail. We make the following contributions [33,34]:

- Contract-based data structure repair: We introduce the idea of using rich behavioral contract specifications, including invariants, preand post-conditions, as the basis of systematic data structure repair.

- Repair algorithms: We present four algorithms that embody the idea. The algorithms leverage the Alloy tool-set to provide systematic repair, and employ heuristics to optimize performance.

- Similarity metric: Our approach uses a distance metric for graph similarity to compute the effect of repair on an erroneous program state and to evaluate different algorithms for effectiveness. 
- Evaluation: We present experimental results to evaluate the feasibility of contract-based repair and demonstrate the promise it holds. We evaluate Tarmeem using a text-book data structure and an open-source application. Our framework can repair erroneous data structures with tens of nodes in seconds.

The rest of this document is organized as follows: Chapter 2 describes an example which we use for further illustration. Chapter 3 provides needed background on the Alloy tool-set. Chapter 4 explains Tarmeem, Chapter 5 presents our experimental results and Chapter 6 concludes. 


\section{Chapter 2}

\section{Example}

To illustrate contract-based repair, consider a singly-linked list data structure (Figures 2.1 and 2.2). As Figure 2.1 shows, each list object has a header node and a size field that caches the number of nodes in the list. Each node has a next node and contains an integer element (elt). The method contract provided in JML shows that the rep0k method (Figure 2.1) is the class invariant. The method remove (Figure 2.2) removes all occurrences of the given integer $(\mathrm{x})$ from the given list (this). This method requires the class invariant to hold at the invocation of the method and should guarantee the class invariant as well as the post-condition after execution.

The user provides the contract including the class invariant for LinkedList and the post-condition for remove in Alloy (Figure 2.3) or any other specification language that can be automatically translated to Alloy such as JML (Figures 2.1 and 2.2). This flexibility in terms of input specification language enhances the usability of Tarmeem. However the specification which is used as a basis for repairing erroneous outputs is eventually translated to Alloy. Chapter 3 provides a brief survey of Alloy syntax and semantics. In this example, the class invariant requires that the list should be acyclic, contain unique inte- 


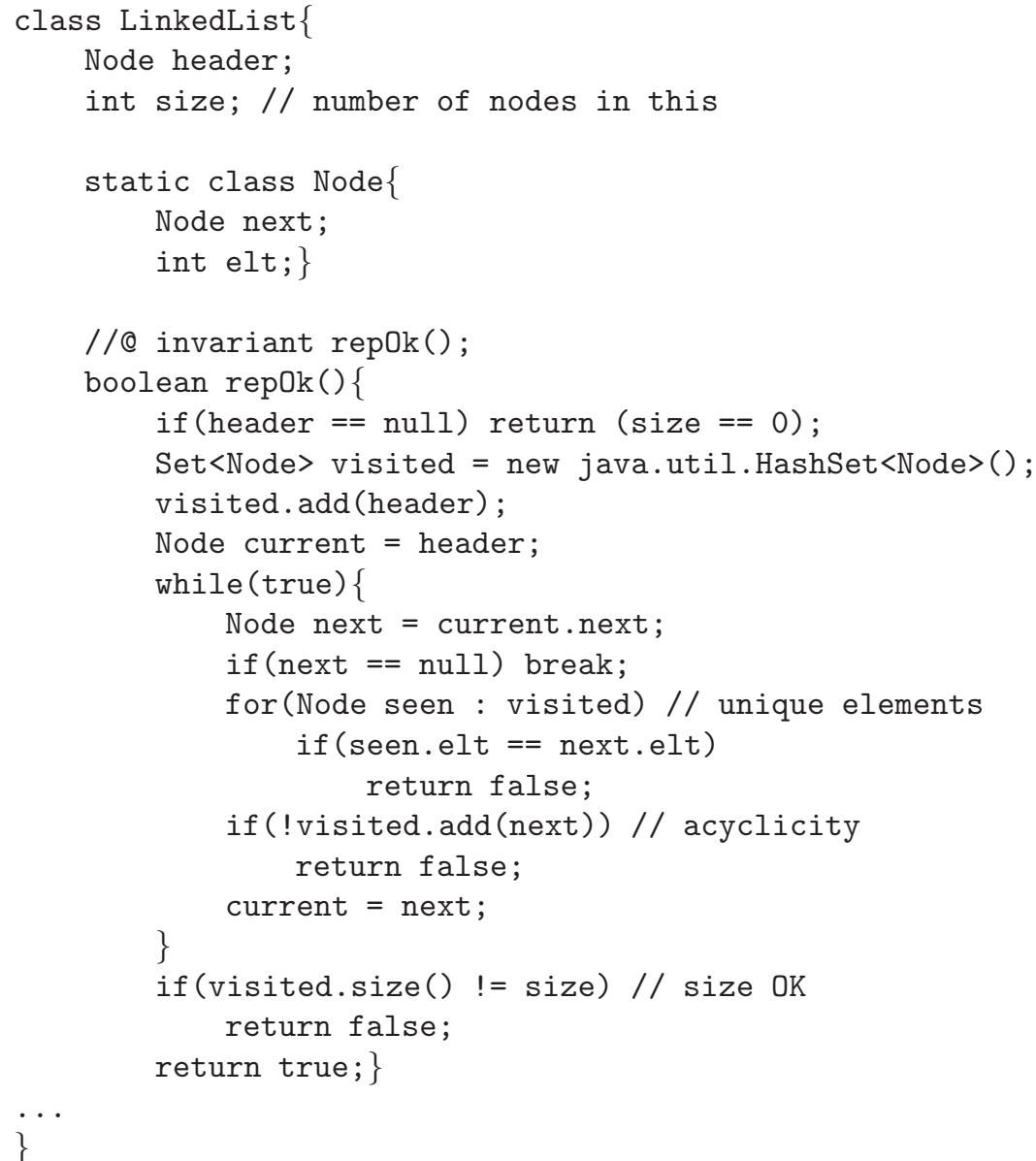

Figure 2.1: Singly-linked list in Java. 


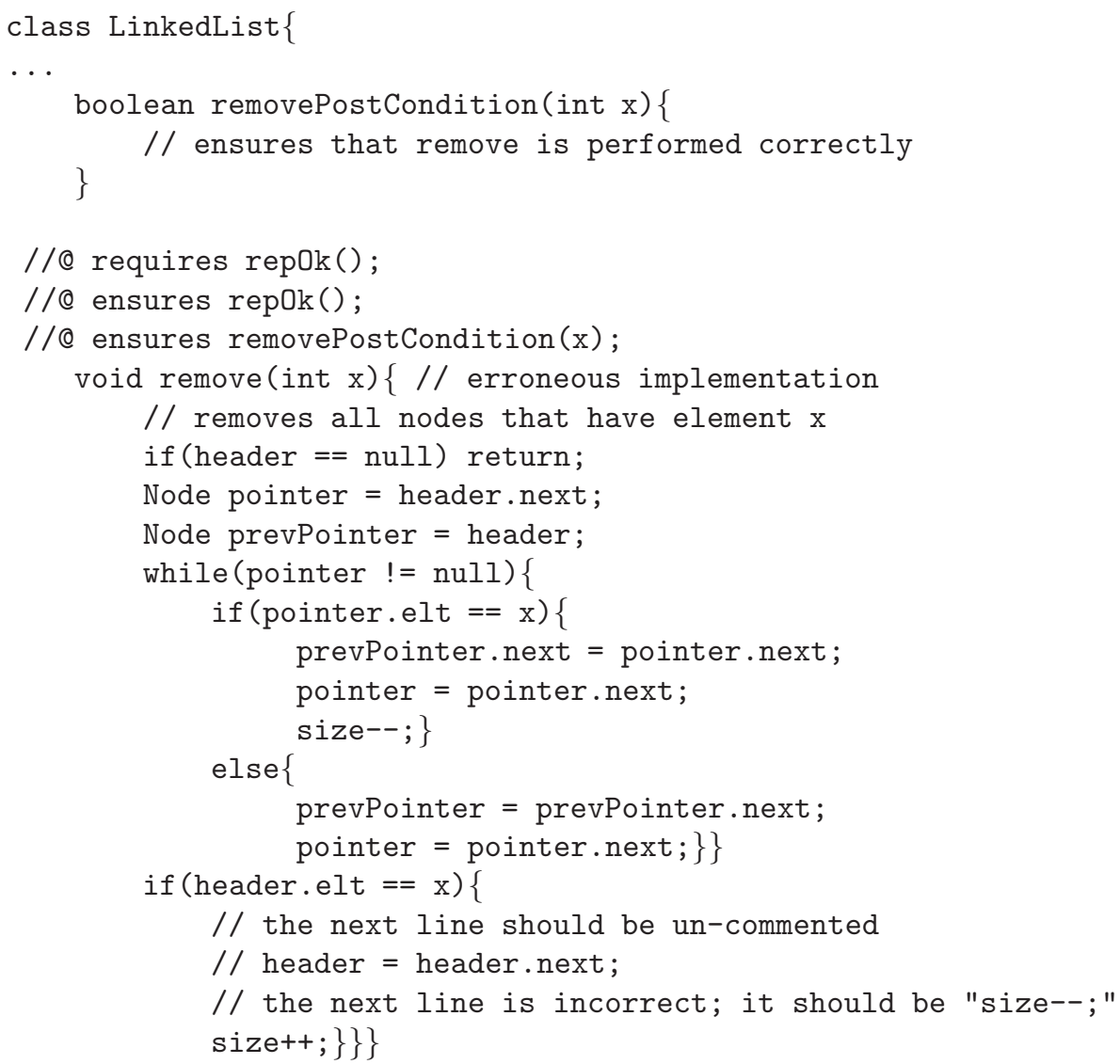

Figure 2.2: An erroneous remove method for singly-linked list in Java.

ger elements, and have correct size. The post-condition additionally requires that the output should not include the element to remove. Back-tick (',') in the Alloy model is syntactic sugar to represent post-state [17,25]. Equivalently absence of back-tick is the same as \old in JML. Note how the post-condition relates the set of list elements in the post-state with those in the pre-state to precisely specify correctness of remove.

To illustrate Tarmeem, consider invoking remove on the valid input 


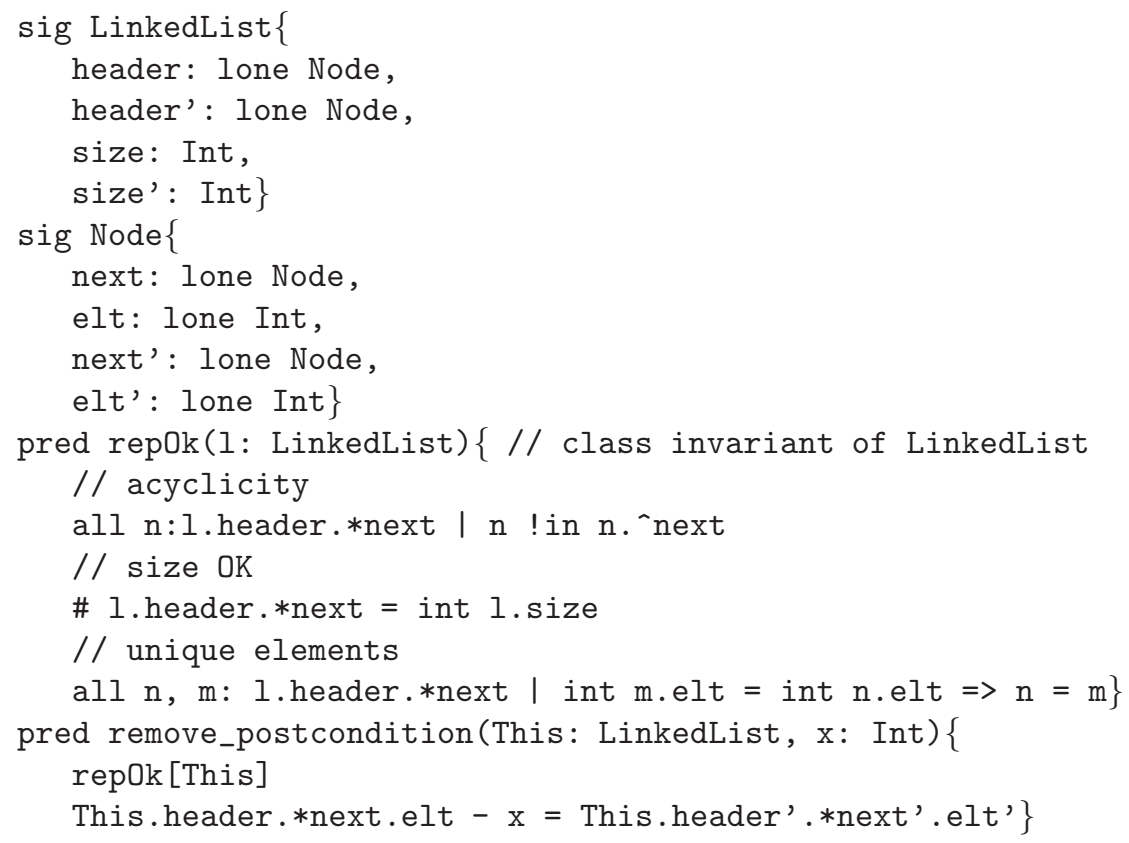

Figure 2.3: Class invariant for LinkedList and post-condition for remove in Alloy.

list Lo in Figure 2.4 (a) to remove all nodes with element 5. Figure 2.4 (b) shows the method's erroneous output list, which has an erroneous size and still includes the element 5. Tarmeem correctly repairs this erroneous output to a correct output (Figure $2.4(\mathrm{c})$ ). Note that Tarmeem corrected both the erroneous data field as well as the erroneous reference fields.

To contrast with the previous work $[10,18]$, if we only use the class invariant (repok) as a basis of repair, the erroneous output list (Figure 2.4 (b)) would only undergo one repair action, which is to fix its size to 3. However, the element 5 would erroneously continue to be in the list, and not be deleted. 

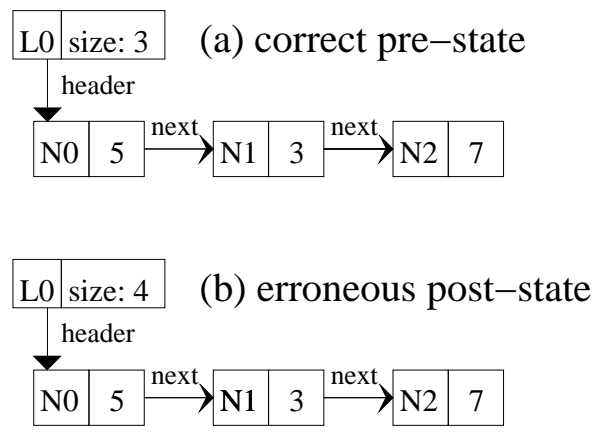
\begin{tabular}{|l|l|l|}
\hline L0 & size: 2 (c) repaired post-state
\end{tabular} header
\begin{tabular}{|l|l|l|l|}
$\mathrm{N} 1$ & 3 \\
\hline
\end{tabular}

Figure 2.4: Linked list example. (a) header $=\{(\mathrm{LO}, \mathrm{NO})\}$ size $=\{($ LO, 3) $\}$ next $=\{(\mathrm{NO}, \mathrm{N} 1),(\mathrm{N} 1, \mathrm{~N} 2)\}$ elt $=\{(\mathrm{NO}, 5),(\mathrm{N} 1,3),(\mathrm{N} 2,7)\}$

(b) header $=\{(\mathrm{LO}, \mathrm{NO})\}$ size $=\{(\mathrm{LO}, 4)\}$ next $=\{(\mathrm{NO}, \mathrm{N} 1),(\mathrm{N} 1, \mathrm{~N} 2)\}$ elt $=\{($ NO, 5), (N1, 3), $(N 2,7)\}$

(c) header $=\{(\mathrm{LO}, \mathrm{N} 1)\}$ size $=\{(\mathrm{LO}, 2)\}$ next $=\{(\mathrm{N} 1, \mathrm{~N} 2)\}$ elt $=\{(N 1,3),(N 2,7)\}$

Figure 2.5: Relational representation of the linked list example. 


\section{Chapter 3}

\section{Background}

Alloy is a minimal yet powerful specification language for describing structural properties. Alloy uses a set-based syntax which is compatible with an object-oriented model. Alloy Analyzer is a fully automatic analyzer to systematically check Alloy models for a finite scope and is based on a SATbased model finder called Kodkod [30]. In this chapter, we use the linked list example of Figure 2.3 to describe Alloy syntax and semantics. More details may be found elsewhere $[1,14]$.

The model is divided into signatures indicated by keyword sig. LinkedList is a signature in this example whose body includes header, header', size and size'. From object-oriented perspectives, these could be considered fields of data type LinkedList with their types following the colon (:) symbol. For example, header is of type Node defined later. Since Alloy is a declarative rather than an imperative language, the order of elements is irrelevant. Keyword lone can be read as less than or equal to one, which means that a LinkedList is permitted to have no header.

From Alloy's set-based perspective which views the world as a collection of atomic objects (atoms) and relations between them, each of header, header', 
size and size' is a relation from signature LinkedList to the signature type following the colon symbol. Figure 2.5 shows realizations of these relations. Consequently, lone and similar keywords have a set-based meaning (e.g. Ione indicates that the relation is a partial function).

A fact (fact) in the Alloy model puts some always true explicit constraints on the model. Functions (func) and predicates (pred) are other forms of constraints to be applied on the model which are invoked if needed. Here, predicate repok has input parameter 1 of type LinkedList surrounded by parenthesis. Note that at the invocation site, brackets are used to pass the arguments. Predicate rep0k is composed of the conjunction of several constraints. For example, the first constraint enforces acyclicity on the list. The $\operatorname{dot}($.$) operator is relational composition, which accesses fields of a signature,$ where $*$ and - are reflexive and non-reflexive transitive closures respectively. 1.header.*next builds the set of all nodes that can be reached by applying zero or more next relations on 1. header which are exactly all nodes of the list. The universal quantifier all on the right-hand side of this constraint indicates that the constraint applies to all of the atoms in the set. The left-hand side specifies that no node $\mathrm{n}$ in the list can reach itself by applying one or more next operations, i.e. the list is acyclic.

Further constraints of this example introduce other features of Alloy. Alloy supports basic set operations. The operators in, \# and - are set membership, cardinality and complement operations respectively. Alloy also has different types of logical binary operators. As an example, => in uniqueness 
constraint stands for implication. Notice that $=$ in Alloy stands for equality and not assignment. As we mentioned before, back-tick is the syntactic sugar we add to Alloy to distinguish between pre- and post-states and is not inherent to the language.

Kodkod is the SAT-based back-end of the Alloy Analyzer. It is a constraint solver which works in first order logic with relations, transitive closure, and partial models. Kodkod is able to efficiently find finite models for satisfiable problems and a minimal unsatisfiable core for unsatisfiable ones. It also provides an API to directly build Alloy formulas. To model a problem in Kodkod, a relation should be bounded by two sets of tuples: a lower bound and an upper bound. The former represents all tuples that the final solution for the relation should contain and the latter is the set of all tuples that it may contain. If a relation is bounded exactly to a set (equivalently its lower bound is the same as its upper bound), that set is the unique answer for the relation.

To illustrate how Kodkod works, consider Figure 2.5 (b) which shows the relational representation of Figure 2.4 (b). Assume that we are trying to repair this data structure to meet the constraints of Figure 2.3. For the sake of simplicity, assume that we do not care about remove_postcondition and our goal is to transform the faulty linked list to a correct one irrespective of being the output of remove method. If we fix the lower and upper bounds of the relations to tuples shown in Figure 2.5 (b) and call Kodkod to find a satisfiable solution for given constraints, Kodkod will have only one candidate which is the same as the faulty output and does not meet the constraints. However, 
if we set the lower bound of the size relation to the empty set and make its upper bound equal to the set of all possible tuples that show the size of a linked list (i.e. $\{\ldots,(\mathrm{LO},-2),(\mathrm{LO},-1),(\mathrm{L} 0,0),(\mathrm{LO}, 1),(\mathrm{LO}, 2), \ldots\})$, Kodkod will provide the correct set which is $\{(\mathrm{L} 0,2)\}$ as answer. Note that this example was used to illustrate the concept of lower an upper bounds in Kodkod and does not contain the most important contribution of this work which is satisfying the relationship between pre- and post-states of a method. 


\section{Chapter 4}

\section{Tarmeem}

This chapter first defines the repair problem (Section 4.1) and then presents an overview of our framework (Section 4.2) and our core repair algo-

rithms (Section 4.3). It finishes by describing more underlying details of the framework and demonstrating preparation steps that instrument the program to use data structure repair (Section 4.4).

\subsection{Definition of repair}

We address the following repair problem:

Definition: Let $\phi$ be a method post-condition that relates pre- and post-states such that $\phi(r, t)$ if and only if pre-state $r$ and post-state $t$ satisfy the post-condition. Given a valid pre-state $u$, and an invalid post-state $s$ (i.e., $! \phi(u, s))$, mutate $s$ into state $s^{\prime}$ such that $\phi\left(u, s^{\prime}\right)$.

The key technical difference in the repair problem as defined here with previous work $[10,18]$ is to allow specifications across different program states, specifically to relate pre-states and post-states. Previous work only supported specifications on the current program state, e.g., class invariants. With this richer definition, we can also repair program functionality error such as incor- 
rect implementation of a method by fixing the output during program execution.

\subsection{Overview}

Tarmeem, our contract-based data structure repair framework, detects and fixes erroneous method executions using method post-conditions. Upon detecting a violation, Tarmeem uses heuristics to determine which fields of the data structure are likely faulty. It iteratively relaxes bounds on possible values for those fields (i.e., allows them to take any legal value other than their current one), and invokes off-the-shelf SAT solvers to compute values that satisfy the post-condition. Furthermore, Tarmeem heuristically tries to keep the number of mutations applied on the faulty data structures as low as possible to avoid unnecessary perturbations during the repair process.

We take a relational view of the program heap [16], and view the data structures as edge-labeled graphs. This view enables using edit distance [8,29] as a metric for computing the perturbation of the erroneous program state, which undergoes repair. Our algorithms attempt to keep the perturbation to a minimum.

The state of the program is defined as an object graph of its data structures. As an example, we have previously shown the state of a program that only includes a linked list of nodes in Figure 2.4. By using such object graphs, we utilize the edit distance of the faulty and repaired outputs to eval-

uate effectiveness of repair algorithms. We heuristically try to minimize the 


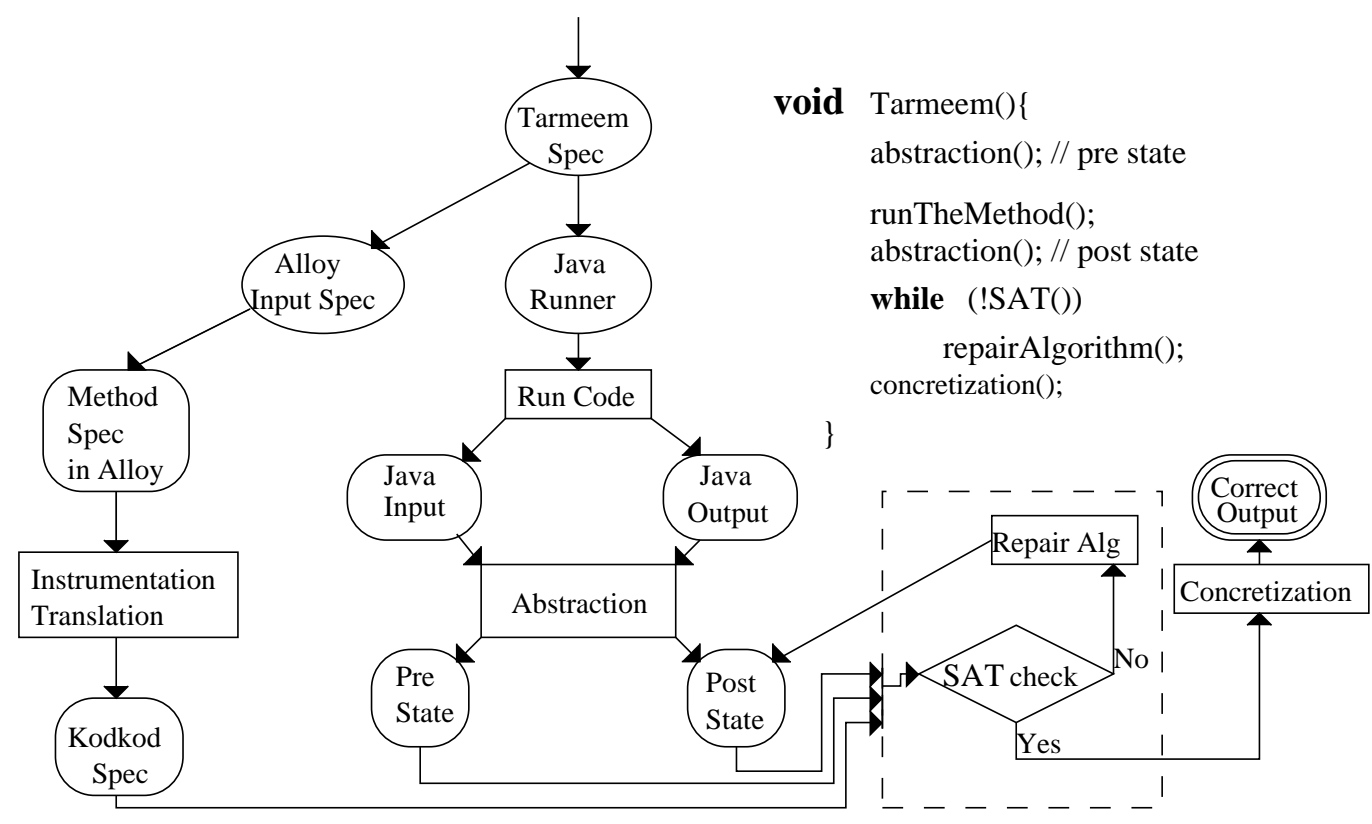

Figure 4.1: Tarmeem architecture.

edit distance between faulty and repaired outputs and measure the amount of perturbation using this metric.

Figure 4.1 shows the core architecture of Tarmeem. Given a Java method and its contract, plus an input and an output that represent an erroneous method execution, Tarmeem applies an abstraction translation [17, 25] to generate an abstract ${ }^{1}$ pre-state and an abstract post-state, which are used together with a constraint that represents the post-condition to check if the post-condition holds. If the pre/post-states satisfy the post-condition, a concretization translation $[17,25]$ is used to generate a repaired output. Note

\footnotetext{
${ }^{1}$ Here, abstraction is to translate the Java data structures to Alloy and concretization is to translate back to Java.
} 
that the very first check of the method output to see if it is faulty does not need an invocation of the SAT solver. Furthermore, even though the translation of the method input and output to/from their corresponding abstraction in Alloy is done via instrumentation and is very fast, one can perform the first check solely as an assertion check to avoid the performance overhead of abstraction after each method call. If the pre/post-states do not satisfy the post-condition, Tarmeem invokes a repair algorithm (Section 4.3) to let some fields of the data structure be modified by the SAT solver (by relaxing the lower and upper bounds in the corresponding Kodkod model on the ranges of field values in the post-state) and uses SAT to compute values for those fields (while holding the pre-state values constant ${ }^{2}$ ). More details about the architecture of Tarmeem can be found in Section 4.4. The next section describes our repair algorithms.

\subsection{Algorithms}

We developed four different approaches to repair a faulty data structure.

1. Basic Repair is the direct application of SAT to generate a correct output for a method.

2. Iterative Relaxation leverages the fact that there is a relatively few number of errors in the data structure and tries to find a repair by iteratively

\footnotetext{
${ }^{2}$ Tarmeem follows the usual meaning of class invariants, and method pre/post-conditions: an incorrect method execution is defined as satisfaction of pre-condition and violation of post-condition.
} 
relaxing the lower and upper bounds of the relations.

3. Error Localization concentrates on the specification to precisely locate the errors and fix them.

4. Guided Error Localization uses the same approach as Error Localization, but utilizes repair guides provided by the user.

This section describes our repair algorithms in details.

1. Basic Repair. This approach is a direct application of SAT to generate a post-state to satisfy the post-condition. This approach uses the pre-state but is oblivious of the erroneous post-state. Any possible correct output with respect to the contract is a candidate solution for this approach. Therefore, the repaired state may be quite different from the erroneous state. In fact, this algorithm only uses the contract and ignores the code that is already developed and extensively tested. To illustrate, consider the remove method (Chapter 2) that should remove all occurrences of the given element from the given list. According to the first approach, any linked list that only has all elements other than the element to delete is a valid answer. This approach does not need to maintain order of elements or other characteristics of the list, although the program whose output is faulty and subject to repair might be intended to maintain these characteristics.

Before describing the algorithm, here we define the state of being relaxed for a relation to ignore the current (possibly erroneous) value of that field 


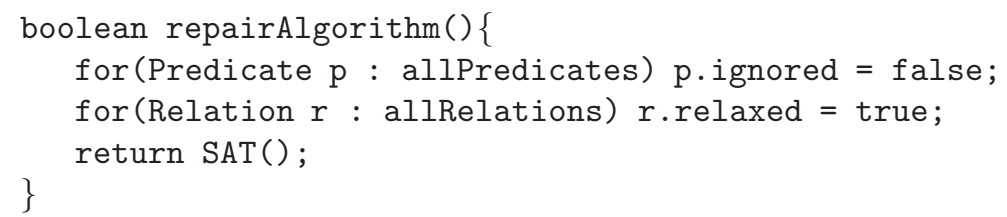

Figure 4.2: Approach 1: the Basic method.

(relation in set-based perspective of Alloy). Therefore, a relaxed field can take any value suggested by the SAT solver to meet the constraints. In addition, we define the property of being ignored for a predicate ${ }^{3}$. We manipulate this property of the predicates to ignore them in some phases of the algorithms. Essentially, we set a predicate to be ignored in case we do not want to include it in a SAT solver invocation.

Figure 4.2 describes an algorithm that implements this approach. It relaxes the bounds on all relations (in the post-state) as indicated by the assignment $r . r e l a x e d=$ true, and solves the problem subject to all the constraints as indicated by the assignment $\mathrm{p}$.ignored $=$ false. The method $\operatorname{SAT}()$ simply calls the SAT solver with these settings and returns true if the SAT solver can find a solution.

Figure 4.3(b) shows a result of running the first approach on the faulty output of our example shown in Figure 4.3(a). The result has changed the

\footnotetext{
${ }^{3}$ Remember that predicates are constraints on the correct output and their conjunction builds the post-condition and the invariant. Predicates used in the repair algorithms might have more granularity compared to Alloy predicates mentioned before, meaning that we might choose to pick each property of the correct output (such as acyclicity, size, etc.) as a single predicate in order to improve performance and accuracy of the repair process.
} 


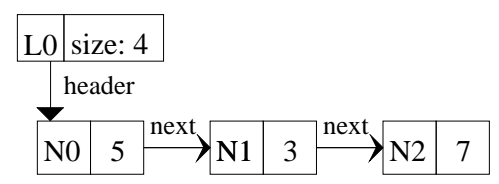

(a)

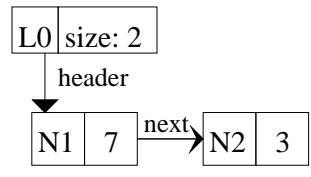

(b)

Figure 4.3: (a) The erroneous output of remove (5) (b) The result of repairing the sample faulty output using Basic approach.

order of elements and assignment of integers to the nodes. Also, as we will see in Chapter 5 this approach is quite inefficient. However, note that this is a correct repair, since the repaired linked list satisfies the method postcondition with respect to the contract. Indeed, a richer contract that requires the relative element ordering to be preserved would result in a more desirable repaired structure using this approach.

2. Iterative Relaxation. The second approach, called Iterative Relaxation, aims to optimize performance when the number of errors is relatively small. In this heuristic we try to make the output satisfy the conditions by relaxing as few relations as possible. To do so, we take an arbitrary relation, relax it and send the resulting problem to SAT. If the problem is unsatisfiable, we take another relation and repeat the same procedure. If we are not able to solve the problem by relaxing a single relation we try relaxing two relations and so forth. If the underlying repair problem is satisfiable, this approach will solve it, but may need a lengthy trial and error process. In addition, this heuristic will find a relatively similar answer to the faulty output since it prefers relaxing fewer relations. 


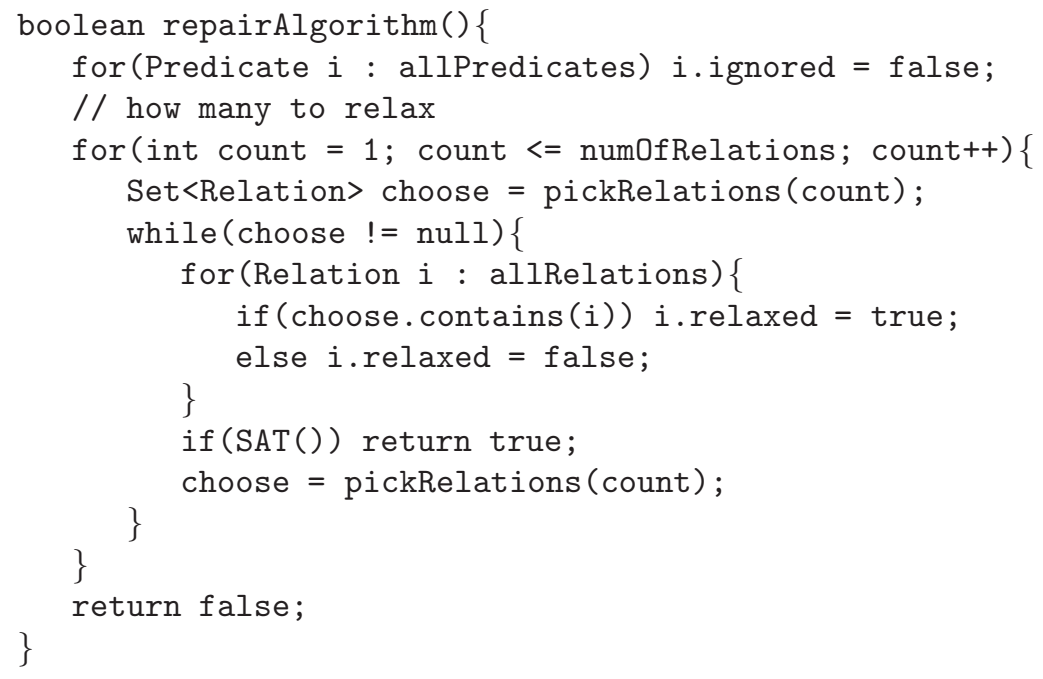

Figure 4.4: Approach 2: Iterative Relaxation.

The pseudo code of this heuristic is shown in Figure 4.4. Having all predicates in mind, we increase count starting from one. pickRelations (count) returns a new arbitrary set (of size count) of relations until all combinations are covered when it returns null. We fix all relations except those selected for relaxation to their post-state values. Each time we call SAT() to see if we have been able to resolve the problem. Upon failure, we examine other combinations of relations while trying to keep them few until we find an answer or confirm that there is no solution.

Back to removing an element from the linked list, we start by taking a relation, say size and let it be free to take any value assigned by the SAT solver. Then we invoke the SAT solver to see if it can find a suitable value for size in order to satisfy all conditions. Clearly, this trial fails since there is an error in the elements too. We attempt relaxing header, next and elt 


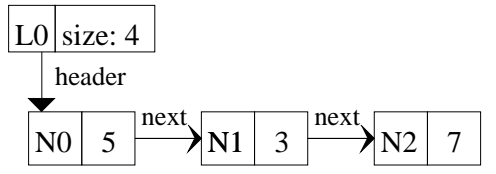

(a)

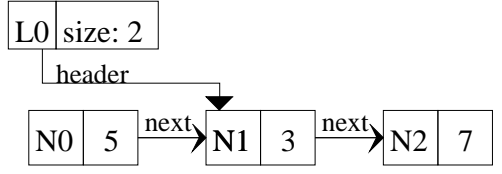

(b)

Figure 4.5: (a) The erroneous output of remove (5) (b) The result of repairing the sample faulty output using Iterative Relaxation, Error Localization and Guided Error Localization.

separately, none of them ending in a solution. When up to relax two relations instead of one, we will finally try size and header together. The result of this repair action (applied on Figure 4.5(a)) is shown in Figure 4.5(b).

3. Error Localization. In the third approach, termed Error Localization, we use the post-condition to isolate erroneous parts of the output. The idea is to satisfy predicates of the post-condition one at a time ${ }^{4}$. For each violated predicate, we relax some of its relations until it is satisfied. Therefore, this algorithm heuristically avoids relaxing relations that do not appear in violated predicates.

At first, we ignore all post-condition predicates and find a solution that is compatible with the definition of data structure. Definition of a data structure is the set of its signatures and does not include class invariants or method post-conditions. (As an example of data structure definition, consider Figure 2.3 without rep0k and remove_postcondition). Then, we evaluate pred-

\footnotetext{
${ }^{4}$ We assume that the post-condition is the conjunction of several predicates. (Examples of these predicates in Figure 2.3 are correctness of the size field and absence of the element subject to remove after calling remove method.)
} 
icates one by one. (Note that to simply evaluate a predicate, no call to the SAT solver is necessary.) If the solution is already meeting the current predicate, we add this predicate to the set of already satisfied predicates and continue by considering others. Otherwise, we should call the SAT solver to find a satisfiable solution for the set of predicates seen so far, while possibly relaxing more relations.

More detailed description of Error Localization heuristic is as follows. Figure 4.6 called with guided set to false shows this heuristic. First, we ignore all predicates and let all relations be not relaxed. An ignored predicate would not participate in solving the problem as it does not exist for now. (These predicates will be added and satisfied eventually.) We call the SAT solver with these settings and expect to obtain a satisfiable answer unless there is a contradiction in the output even without applying any constraints. Having this satisfiable answer assists us in evaluating further constraints as we add them without going to the SAT solver level (by using Evaluator class at the Kodkod level).

While there is any ignored (i.e, not satisfied) predicate, we add predicates and try to fulfill them. Note that at the beginning of each iteration of the while loop, we check all predicates to find out if any of them are satisfiable on our current answer. If any is found, we add it to the set of satisfied predicates by setting ignored to false and updating relations seen. The sooner we add satisfied constraints, the easier it is for SAT to find the correct value.

At the next step, we choose an arbitrary predicate which is not satisfied 


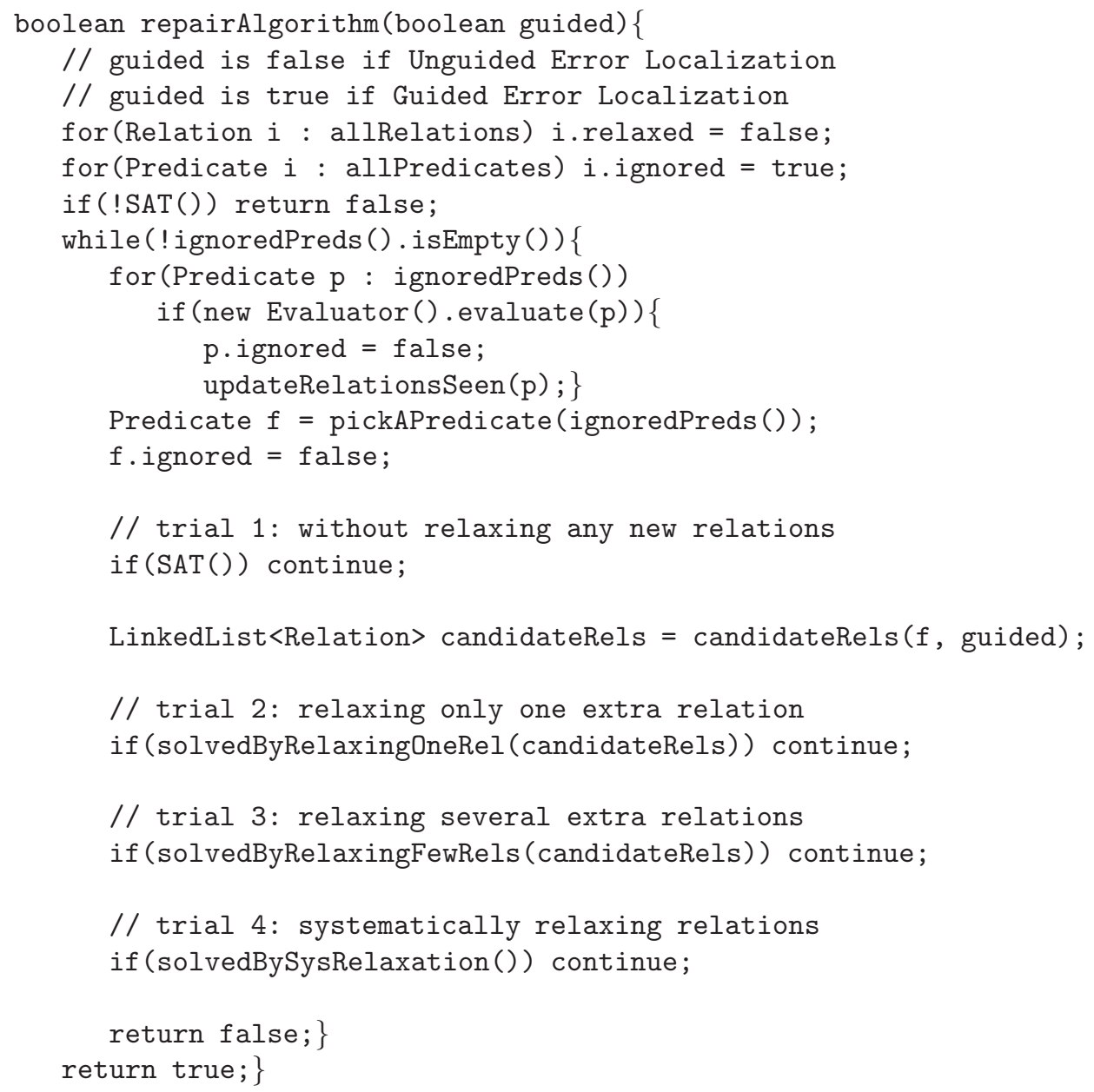

Figure 4.6: Approaches 3 and 4: Error Localization.

yet and attempt to satisfy it by relaxing some extra relations. We watch the previous constraints so that we do not break them by sending all predicates added so far to the SAT solver.

Our trials to satisfy the current predicate proceed as follows:

1. Our first priority is to satisfy the predicate without relaxing any new 


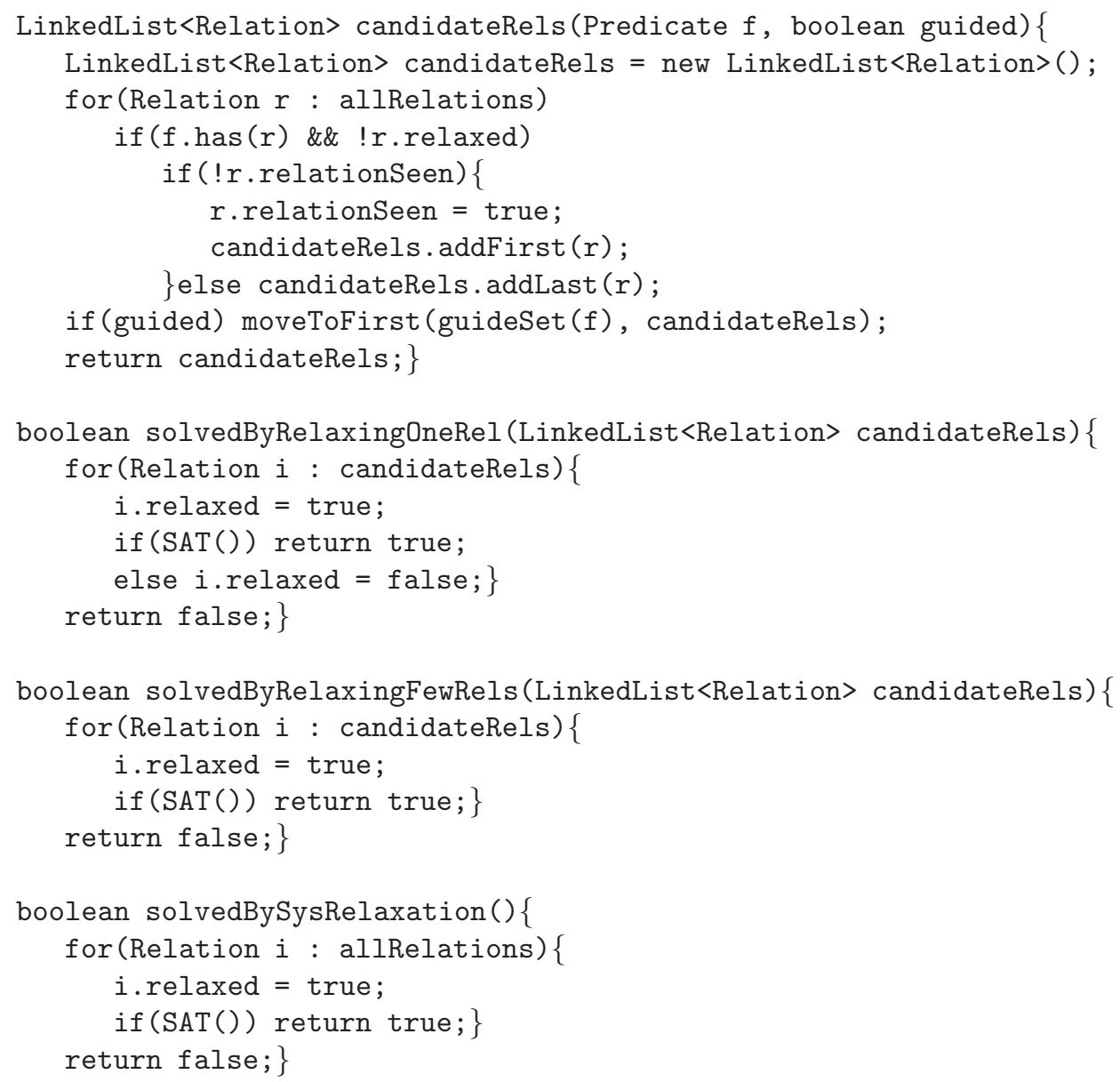

Figure 4.7: Approaches 3 and 4: helper methods.

relations. Note that this is different than applying the predicate on current instance; here we call SAT to see if we can find another instance with the same relations relaxed that satisfies the new predicate as well.

2. Then, we try to meet the predicate by relaxing only one extra relation prioritizing those which appeared in this predicate for the first time (method solvedByRelaxingOneRel in Figure 4.7). The list of non-relaxed 
relations of this predicate is constructed in candidateRels linked list. In this list, relations seen for the first time (i.e, not used in the previous predicates) come first. Method candidateRels() (Figure 4.7) called with guided set to false generates this list for each predicate. We intuitively prefer to start by relaxing relations which are new since the already seen relations have been used in previous constraints and have shown no sign of violation.

3. If the predicate is still unsatisfied, we relax several extra relations by invoking method solvedByRelaxingFewRels in Figure 4.7. We accumulatively relax relations, meaning that we relax a set of relations as we go along candidateRels list.

4. If we were not able to resolve the problem despite relaxing all relations of this predicate, it means that we have made a mistake somewhere in previous predicates. So, by calling method solvedBySysRelaxation in Figure 4.7, we systematically relax other relations until we can satisfy the current constraint.

If all of the above trials ${ }^{5}$ fail on relaxing a specific predicate, the method returns false. Otherwise it returns true after satisfying all predicates.

Consider the faulty linked list output of the remove method once again (Figure 4.5(a)). At the first iteration, we realize that constraints uniqueness

\footnotetext{
${ }^{5}$ We also experimented with backtracking to fix previous wrong relaxations, but this approach was not feasible with respect to performance.
} 
and acyclicity are holding without any relaxations. Assume that we continue the loop by trying to satisfy the size constraint. The first relation in candidateRels list will be size since it is the only new relation of the size constraint (next and header are already seen in acyclicity and uniqueness). By relaxing size, we will be able to meet this constraint and move to the next one. The next constraint is correct remove. Candidate relations would not prefer any of un-relaxed relations over the others since header, next and elt are all seen before. At the worst case the algorithm tries next and elt before header but once it gets to header (in addition to already relaxed size), all constraints would be satisfied and we obtain Figure 4.5(b) as repaired output. Note that when we first fixed size, we set it to 3 since it was the correct list size at that time. However, when we relax header, this value of size is not acceptable any more. Yet, there is no need to go back since size is still relaxed and the SAT solver will find an acceptable value for it as well.

4. Guided Error Localization. While evaluating repair heuristics, we found that none of them can accurately guess which relation is most likely to be the cause of violating a specific predicate. So, we let the user guide the repair mechanism by indicating which relation is subject to evaluation in each predicate. For each predicate, the user indicates an ordered list of data structure parameters, called the guide set, which are intuitively tested under this predicate. To illustrate, consider the constraints of Figure 2.3. Constraint acyclicity mainly checks the next pointer to detect cycles so its guide set starts 
with next. However, it does not evaluate header although it uses header as well. Therefore, header is either not included in the guide set or has less priority. In this figure, constraint size is to check size relation, uniqueness evaluates elt, and correct remove deals with next and elt. Including the guide set in the method contract requires adding JML annotations to indicate members of the guide set for each ensures statement (for example in Figures 2.1 and 2.2).

Note that providing the guide set is an easier task compared to writing a flawless method. Indicating that the output of a method should satisfy some predicates comes directly from the method specification. Similarly, relations that are important in any predicate come from programmer's understanding of the predicate and are not tied to any specific method implementation. While using these guides appropriately will help the performance of the repair process considerably, there is no syntactic restriction on the user. In some cases, we can even tolerate wrong guide sets. The guide set is only a hint to prioritize relaxations and the search can revert to an unguided mode if necessary.

To provide the guide set, the user indicates relations tested in each constraint. For example, if we added next to the guide set of the uniqueness constraint, we would help the repair method's performance. However, we did not assume the existence of the best guides in our evaluation.

Figure 4.6 together with Figure 4.7 when guided set to true shows the guided version of Error Localization. The only improvement over the unguided approach is moving the guide set to the beginning of candidateRels (Figure 4.7), so that they will be checked sooner, protecting the repair process 


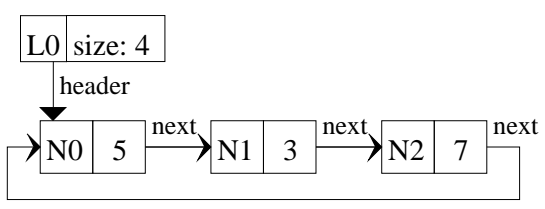

(a)

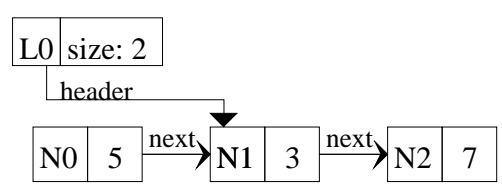

(b)

Figure 4.8: (a) Circular linked list: a hypothetical erroneous output of remove(5) (b) The result of repairing the faulty output using Guided Error Localization.

from unnecessary calls to the SAT solver.

Applying this approach on our example (Figure 4.5(a)) might possibly go through exactly the same process (to provide Figure $4.5(\mathrm{~b})$ ) as it did for unguided repair since header is not among the guide set of correct remove. However, the difference shows up when we want to repair the data structure of Figure 4.8(a) as erroneous output. The only satisfied constraint here is uniqueness. Assume that we choose to repair acyclicity first. Since next and header are both seen before, regular Error Localization does not prioritize any of them. Yet, a good guide set which indicates that the most likely error in breaking acyclicity is in next will save us trying header to meet acyclicity. The repaired data structure is shown in Figure 4.8(b).

It is worth noticing that for our simple example, all approaches besides the Basic one provide the same output for both Figure 4.5(a) and Figure 4.8(a), which means that all these three approaches are optimized to keep the mutations as low as possible, though they face the restriction of whole relation relaxation instead of edge correction. A differentiation here is which method provides the result faster with fewer SAT solver invocations. Table 4.3 com- 
Table 4.1: Comparison of different repair Algorithms.

\begin{tabular}{|l|c|c|c|}
\hline & Uses post-state & Uses post-condition to optimize & Uses annotations \\
\hline Basic approach & & & \\
\hline Iterative Relaxation & $\sqrt{ }$ & $\sqrt{ }$ & \\
\hline Error Localization & $\sqrt{ }$ & $\sqrt{ }$ & $\sqrt{ }$ \\
\hline Guided Error Localization & $\sqrt{ }$ & & \\
\hline
\end{tabular}

pares all four approaches from a high level perspective.

\subsection{Instrumentation}

In this section, we provide more details on Tarmeem underlying structure (See Figure 4.1). These are the steps Tarmeem takes to instrument the Java program and reach the point that the repair heuristics are applicable.

Alloy input specification: Tarmeem represents the data structure in Alloy and uses the technique of duplicating [19] to distinguish between the state of data structure prior to and after the method execution. Figure 2.3 illustrates the data structure, the invariant and the post-condition for our simple example of a linked list and a method which removes an element from it. This is essentially the same data structure we had in Java with header, size, next and elt representing the same fields of the Java program in the pre-state (i.e, before running the method). We use the backtick character (" ') to represent the same filed in the post-state (i.e, after running the method).

Another important aspect of the Alloy model is how to write the constraints. Here we prefer to write the constraints manually since we want to ob- 
tain high level constraints. Also we need more manageable constraints since we sometimes desire to break the constraints to several parts to help the heuristics. In general, these more controllable constraints come with the price of dealing with Alloy predicates manually, though the same concepts can be achieved using methods of [19]. In addition, note that method pre- and post-conditions might already be written in Alloy for testing proposes as described in [19], or in JML as in [7]. In the former case, data repair is provided for free using Tarmeem since the Alloy specifications are already written, and in the latter we can use tools that translate JML specifications to Alloy automatically [32].

Abstraction and concretization: Once the specifications are built, the next step is to keep track of the data structure in pre- and post-states. To store the state of the program, we demonstrate the object graph using relations. A part of the framework is dedicated to keeping track of the data structures. This part will be modified by the user to map the Java data structure to its Alloy representative. The user indicates the mapping intended between the Java data structures and Alloy signatures. This manual configuration can be skipped too using techniques described in [19]. Once the part responsible for translating the data structure to Alloy and Kodkod is constructed, the program can traverse data structures automatically and translate them to Alloy and Kodkod. This is the procedure we called abstraction in Figure 4.1. For each object that appears in pre- or post-states there would be one signature instance in Alloy. Before and after running each method of the program, 
the abstraction part is called to keep track of the object graphs. If an error occurs in the method execution, these object graphs which are represented as relations will be sent to the SAT solver. Concretization does the reverse action of abstraction and translates a repaired data structure back to Java.

During translation of the program state to Kodkod (abstraction), Tarmeem manipulates the Kodkod file to equip it with the relaxed fields; For each relation, a boolean indicates if this relation is relaxed. Also, in abstraction phase all predicates are equipped with the option of being ignored. An ignored predicate will not participate in solving the problem.

Relaxing a relation is the most fundamental concept of Kodkod used by Tarmeem. If a relation is not relaxed, it is bound exactly to its set in the post-state. For example, if the relation next is not relaxed in the linked list example (Figure $4.5(\mathrm{a})$ ), it is set to $\{(\mathrm{NO}, \mathrm{N} 1),(\mathrm{N} 1, \mathrm{N2})\}$ (the value in the post-state). If it is relaxed, its lower and upper bounds will be relaxed and it can take any set that has the correct settings (i.e, maps nodes to nodes) such as $\{(\mathrm{NO}, \mathrm{N} 1),(\mathrm{N} 0, \mathrm{~N} 2),(\mathrm{N} 2, \mathrm{NO})\}$. By relaxation, we mean removing the bounds that are enforced on the relation by the post-condition and letting the output have any legitimate set of tuples for that relation. This means taking out boundExactly from the Kodkod translation, so that instead of imposing the set of tuples provided by the faulty output, we let the relation take any set of tuples ranging from $\emptyset$ to the set of all legitimate tuples. Note that no constraints, even those which indicate the basic definition of the data structure (e.g, there is at most one next for each node as "next: lone Node" states in 


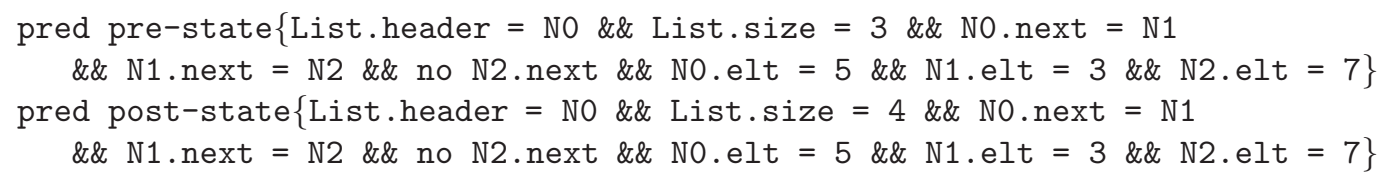

Figure 4.9: Linked List example pre- and post-state snapshots.

Figure 2.3) applies at this level. These type of constraints will be applied later with each SAT solver call.

Run code: When the Java method executes and generates the output data structure, once again Tarmeem collects all objects and assigns Alloy signature instances to them. Then, it takes post-state snapshots using the abstraction part. The translation of Figure 2.4 (a) (input) and Figure 2.4 (b) (faulty output) to Alloy is shown in Figure 4.9. Snapshots are taken by the abstraction part. 


\section{Chapter 5}

\section{Evaluation}

We evaluate Tarmeem using a text-book data structure and an opensource application. Throughout the evaluation, we ran each experiment 5

times and reported the averages. All experiments used a 2.50GHz Core 2 Duo processor with 4.00GB RAM. We used SAT4J [4], a SAT solver in Java which is shipped with Kodkod by default.

\subsection{Singly Linked List}

This data structure is the same as the example singly linked list (Figure 2.3). Each list has a header that points to the first node of the list. Each node has an integer element and a pointer to the next node. The list is acyclic and no duplications are allowed. The list also has a size element which shows the number of nodes.

We implemented several faulty remove methods for this data structure which are described by Table 5.1. The table displays corrupted fields of the output data structure, constraints that are violated by the erroneous remove, and the programmer mistake that can cause such an error.

We ran Tarmeem to fix the output of each incorrect remove method for 
Table 5.1: Faults injected into the programs. Faults 1-7 apply to remove method of singly-linked list, and Faults 8-9 apply to deleteChild method of class BaseTree of ANTLR.

\begin{tabular}{|l|l|l|l|l|}
\hline & Fault name & Erroneous fields & Violates & Program error \\
\hline \hline 1 & Fail to fix the size & size (auxiliary int) & Size & Omission \\
\hline 2 & Delete another element too & next (primary pointer) & Correct remove & Commission \\
\hline 3 & Cycle & next (primary pointer) & Acyclicity & Commission \\
\hline 4 & Broken List & next (primary pointer) & Correct remove and size & Omission \\
\hline 5 & Fail to remove the element & elt (primary int) & Correct remove & Omission \\
\hline 6 & Null header & header (primary pointer) & Correct remove & Commission \\
\hline 7 & $\begin{array}{l}\text { Duplicate an element } \\
\text { and wrong size }\end{array}$ & $\begin{array}{l}\text { elt (primary int) } \\
\text { size (auxiliary int) }\end{array}$ & Uniqueness and size & $\begin{array}{l}\text { Commission } \\
\text { and Omission }\end{array}$ \\
\hline \hline 8 & Fail to delete & $\begin{array}{l}\text { children (primary pointer) } \\
\text { childIndex (auxiliary int) }\end{array}$ & Delete & Omission \\
\hline 9 & Fail to update ChildIndex & childIndex (auxiliary int) & childIndex relations & Omission \\
\hline
\end{tabular}

linked lists of size 10 and also for linked lists of size 20. Equation 5.1 presents the relationship that holds between several different measures with $t$ being the total repair time and alg being the time consumed to do the heuristic algorithm (in sections between calls to the SAT solver). \#SAT_invoc is the total number of SAT solver invocations to repair this specific fault with desired approach and it excludes evaluation calls used to evaluate constraints at the Kodkod level without going to the SAT solver level. SAT_tr avg and $S A T \_s l_{\text {avg }}$ are average translation and solving times over calls to the SAT solver to resolve this specific error. These are measured to give a sense on how SAT translation and solving are time consuming and how a good heuristic which calls the SAT solver fewer times with suitable settings (i.e. relaxations) can be beneficial.

$$
t=a l g+\# S A T \_i n v o c *\left(S A T \_t r_{a v g}+S A T \_s l_{a v g}\right)
$$


Table 5.2: Result of applying Tarmeem on different erroneous singly linked lists of size 10 and 20, and on corrupt BaseTree class of ANTLR package, maximum repair time allowed: 120 seconds.

\begin{tabular}{|c|c|c|c|c|c|c|c|c|c|c|c|c|c|c|c|c|c|}
\hline \multirow{3}{*}{\multicolumn{2}{|c|}{ 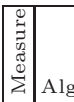 }} & \multicolumn{16}{|c|}{ Erroneous method from Table 5.1} \\
\hline & & \multicolumn{7}{|c|}{ Linked list with 10 nodes } & \multicolumn{7}{|c|}{$\begin{array}{l}\text { Linked list with } 20 \text { nodes } \\
\end{array}$} & \multicolumn{2}{|c|}{ ANTLR } \\
\hline & & 1 & 2 & 3 & 4 & 5 & 6 & 7 & 1 & 2 & 3 & 4 & 5 & 6 & 7 & 8 & 9 \\
\hline (20 & $\mathrm{BM}$ & 7567 & 7314 & 8675 & 3809 & 7629 & 5057 & 5987 & - & - & - & - & - & - & - & - & - \\
\hline ह & IR & 726 & 851 & 1038 & 911 & 883 & 591 & 1764 & 1061 & 9697 & 15784 & 9534 & 2582 & 831 & 5744 & - & 2659 \\
\hline 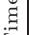 & $\mathrm{EL}$ & 614 & 13113 & 926 & 1160 & 939 & 820 & 1416 & 783 & 25581 & 18622 & 8852 & 2495 & 949 & 4325 & - & 6907 \\
\hline$\vec{F}$ & $\mathrm{GL}$ & 598 & 1060 & 870 & 1031 & 919 & 719 & 1136 & 807 & 14265 & 21180 & 13147 & 2266 & 1240 & 3522 & 13852 & 2196 \\
\hline త్రే & $\mathrm{BM}$ & 38 & 33 & 38 & 33 & 32 & 32 & 34 & - & - & - & - & - & - & - & - & - \\
\hline . & IR & 2 & 12 & 15 & 14 & 19 & 1 & 19 & 2 & 29 & 31 & 28 & 38 & 1 & 39 & - & 10 \\
\hline 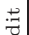 & $\mathrm{EL}$ & 2 & 13 & 15 & 12 & 18 & 1 & 19 & 2 & 26 & 30 & 29 & 38 & 1 & 40 & - & 10 \\
\hline 至 & GL & 2 & 15 & 15 & 14 & 19 & 1 & 22 & 2 & 30 & 30 & 29 & 39 & 1 & 40 & 12 & 10 \\
\hline.$\tilde{\Xi}$ & $\mathrm{BM}$ & 2 & 2 & 2 & 2 & 2 & 2 & 2 & - & - & - & - & - & - & - & - & - \\
\hline$E^{\prime}$ & IR & 4 & 3 & 4 & 4 & 4 & 3 & 10 & 3 & 4 & 3 & 4 & 3 & 3 & 9 & - & 3 \\
\hline & $\mathrm{EL}$ & 3 & 5 & 4 & 5 & 4 & 6 & 6 & 4 & 6 & 5 & 3 & 4 & 4 & 6 & - & 5 \\
\hline \# & $\mathrm{GL}$ & 3 & 4 & 3 & 4 & 4 & 6 & 6 & 4 & 4 & 4 & 3 & 4 & 5 & 7 & 7 & 3 \\
\hline क & BM & 8531 & 8531 & 8531 & 8531 & 8531 & 8531 & 8531 & - & - & - & - & - & - & - & - & - \\
\hline$\stackrel{0}{2}$ & IR & 725 & 1774 & 1421 & 1355 & 2569 & 46 & 2720 & 2175 & 11460 & 15829 & 13620 & 5656 & 146 & 7580 & - & 15824 \\
\hline \# & EL & 320 & 2496 & 1406 & 1518 & 2725 & 351 & 3536 & 432 & 7592 & 17732 & 13300 & 4525 & 100 & 6688 & - & 15564 \\
\hline & $\mathrm{GL}$ & 320 & 1475 & 1645 & 1475 & 2349 & 128 & 1495 & 336 & 15481 & 15510 & 13650 & 4466 & 78 & 5382 & 34359 & 8505 \\
\hline का & $\mathrm{BM}$ & 28836 & 28836 & 28836 & 28836 & 28836 & 28836 & 28836 & - & - & - & - & - & - & - & - & - \\
\hline 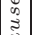 & IR & 2147 & 4855 & 3892 & 3699 & 8828 & 105 & 8995 & 6724 & 34816 & 48196 & 41454 & 19061 & 286 & 24447 & - & 50766 \\
\hline ت ن & $\mathrm{EL}$ & 1106 & 7733 & 3851 & 4384 & 9366 & 1190 & 11989 & 1494 & 23277 & 54082 & 39426 & 15249 & 197 & 21883 & - & 48951 \\
\hline \# & GL & 1106 & 4176 & 4507 & 4176 & 8075 & 406 & 4625 & 1162 & 46354 & 47279 & 40706 & 15048 & 153 & 17263 & 106822 & 28319 \\
\hline क & $\mathrm{BM}$ & 407 & 407 & 407 & 407 & 407 & 407 & 407 & - & - & - & - & - & - & - & - & - \\
\hline 8 & IR & 118 & 100 & 157 & 145 & 209 & 72 & 272 & 179 & 316 & 350 & 316 & 478 & 108 & 597 & - & 807 \\
\hline \# & EL & 21 & 133 & 30 & 75 & 195 & 90 & 311 & 29 & 102 & 162 & 122 & 375 & 220 & 710 & - & 544 \\
\hline & GL & 21 & 48 & 33 & 48 & 185 & 151 & 275 & 22 & 140 & 140 & 130 & 420 & 340 & 683 & 810 & 576 \\
\hline $\begin{array}{l}\text { की } \\
\text { है }\end{array}$ & BM & 320 & 333 & 317 & 319 & 315 & 318 & 346 & - & - & - & - & - & - & - & - & - \\
\hline a & IR & 133 & 173 & 163 & 135 & 169 & 137 & 140 & 259 & 397 & 646 & 395 & 482 & 221 & 502 & - & 503 \\
\hline 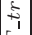 & EL & 128 & 134 & 137 & 112 & 163 & 88 & 154 & 133 & 283 & 525 & 352 & 329 & 176 & 258 & - & 995 \\
\hline $\begin{array}{l}7 \\
0 \\
0\end{array}$ & GL & 124 & 127 & 153 & 123 & 154 & 88 & 128 & 139 & 452 & 512 & 481 & 378 & 200 & 245 & 1579 & 277 \\
\hline है & BM & 3382 & 3242 & 3939 & 1493 & 3420 & 2131 & 2531 & - & - & - & - & - & - & - & - & - \\
\hline a & IR & 4 & 57 & 64 & 59 & 26 & 2 & 15 & 11 & 2080 & 4691 & 2362 & 227 & 3 & 93 & - & 256 \\
\hline$\vec{w}_{1}$ & EL & 4 & 2279 & 58 & 81 & 28 & 5 & 37 & 7 & 3826 & 3592 & 2214 & 167 & 2 & 421 & - & 142 \\
\hline $\begin{array}{l}\vec{k} \\
\infty \\
\infty\end{array}$ & $\mathrm{GL}$ & 4 & 73 & 67 & 73 & 21 & 2 & 11 & 6 & 3035 & 4711 & 3242 & 118 & 1 & 265 & 283 & 243 \\
\hline
\end{tabular}


The edit distance is defined as the minimum number of edge additions or deletions to transform a graph to another and is the same as well-known maximum common subgraph metric under a specific cost function $[8,29]$. Our computation of edit distance is simplified because we have an object graph that is evolved from another one through a sequence of mutations, since the repaired output is an altered version of the faulty output.

Table 5.1 includes experimental results for singly linked lists of size 10 and also 20. \#SAT_invoc represents number of SAT solver invocations, \#vars avg, \#clausesavg and \#pvars ${ }_{\text {avg }}$ stand for the average number of variables, clauses and primary variables in SAT solver calls respectively. SAT_travg and $S A T \_s l_{\text {avg }}$ are average translation and solving times for SAT solver as before. $B M, I R, E L$ and $G L$ are abbreviations of Basic Method, Iterative Relaxation, Error Localization and Guided Error Localization.

In repairing a singly linked list of size 10, while no approach consistently outperforms all others, Iterative Relaxation and Guided Error Localization are the best heuristics. The efficiency of Iterative Relaxation in some cases is because the number of errors in the data structure is assumed to be few and iteratively relaxing relations one at a time will find an error faster if it is only in one parameter. The reason that the Guided Error Localization was not able to defeat Iterative Relaxation in all cases is because in this experiment we preferred to write guide sets in an intuitive fashion. Although such sets are not necessarily optimal, we expect the user to write them as a starting point. In later experiments in Section 5.2 we will see how to write more effective 
guide sets by considering repair process.

The most challenging faulty methods are those which introduce different kinds of errors. For the linked list of size 10, all faulty outputs except the output of erroneous remove number 7 can be repaired in less than 1 second. This method takes at least 1.136 seconds because the error corrupts two parameters of the data structure. However, these types of errors are unlikely in deployed code because it has been systematically tested and also since period checking of post-conditions prohibits propagation of errors in data structures.

In a linked list of size 10, the Basic approach which directly utilizes the post-condition to solve the problem with SAT solver produces some results given sufficient time. However, when we move to 20 nodes, the Basic method can no longer generate the correct output in less than 120 seconds. This shows how applying different heuristics and using the correct parts of the corrupt output can help the repair process. Table 5.1 also presents the result of repairing a singly linked list of size 20. The results are similar to those for a singly linked list of size 10 .

Here, outputs of methods 2, 3, and 4 take more time to repair since they have corrupted the next pointer, a relation which has many possible candidates. One can easily see how solving time of the SAT solver is considerably higher for these three methods. As the data structure size grows, the complexity of the corrupted relation becomes the dominant problem in repair. A possible improvement would be going inside the relation and keeping correct edges instead of relaxing the whole relation, but this solution needs manip- 
ulating the model finder or the SAT solver. Note that even for a linked list of size 20 and with the intuitive guide sets, and the most complicated errors, there is at least one heuristic which can finish the repair process in about 15 seconds or less. The bottleneck is the SAT solver, which takes up to 5 seconds to translate and solve in each invocation.

\subsection{ANTLR BaseTree}

ANTLR: (ANother Tool for Language Recognition [28]), a part of the DaCapo benchmark [6], is a tool that builds recognizers, interpreters, compilers and translators from grammars. ANTLR receives a formal grammar as input and evaluates a given sentence against the grammar. ANTLR uses rich data structures, since it needs sophisticated intermediate forms to recognize and interpret grammars. These data structures are known to be a major source of errors when manipulated incorrectly. This feature makes ANTRL an excellent case study for data structure repair. ANTLR, with 29710 lines of Java code, has a download rate of about 5000 per month and is extensively used to generate programs.

ANTLR represents the grammar internally as a tree. Abstract class BaseTree is a generic tree implementation used to abstract several trees such as ParseTree used in building a parser. ANTLR v. 3 uses a list of children instead of the usual parent child approach in BaseTree. Each tree node maintains a list of children as its successors while each of these children keeps the correct pointer to their parent. Each tree node also keeps integer values startIndex, 


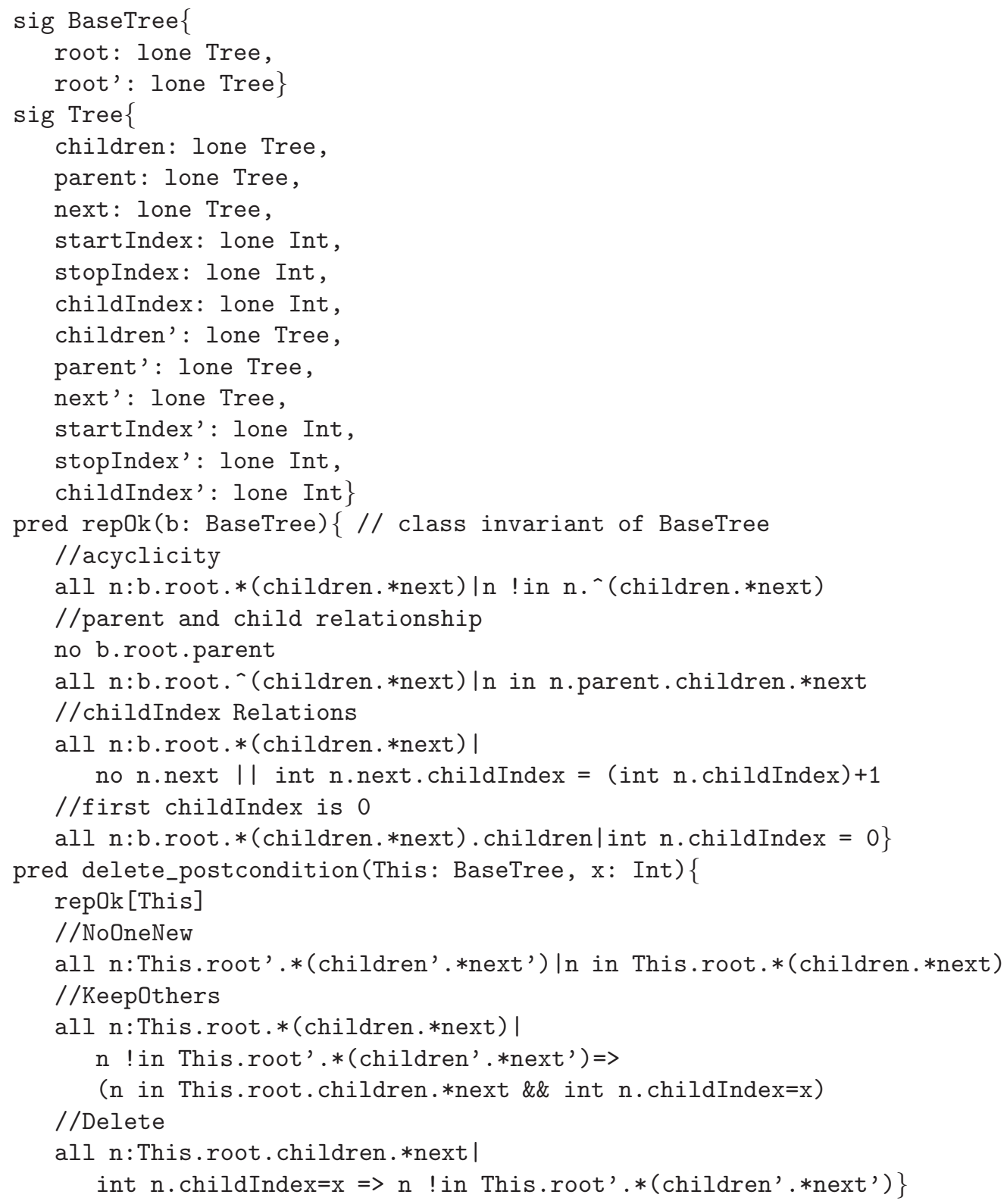

Figure 5.1: Class invariant for ANTLR BaseTree and post-condition for delete in Alloy. 


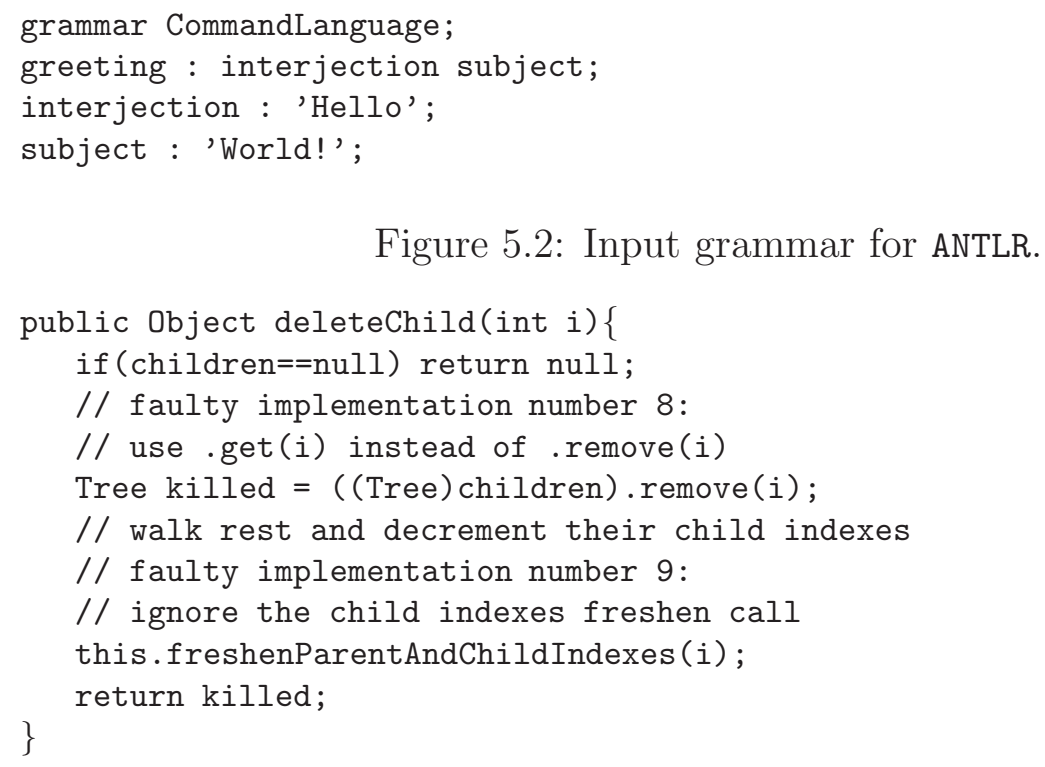

Figure 5.2: Input grammar for ANTLR.

Figure 5.3: deleteChild method of class BaseTree.

stopIndex and childIndex for bookkeeping purposes. For example, childIndex represents the node's place in its parent's children list, counting from zero. In order to be able to use Tarmeem to repair instances of BaseTree data structure, we added class invariants and post-conditions to BaseTree based on comments provided by ANTLR as well as the program logic. The Alloy specification of this data structure is shown in Figure 5.1.

We used ANTLR v.3.1.3 to parse the simple grammar shown in Figure 5.2, which generated 27 instances of class BaseTree in total. Figure 5.3 shows deleteChild method of class BaseTree in ANTLR package with our annotations to inject errors. Table 5.1 demonstrates the result of repairing with Tarmeem. Note that as this table suggests, no heuristic except Guided Error Localization was able to repair the output of error number 8 (See Figure 5.3 
and Table 5.1) in less than 120 seconds. With a good guide set which prioritizes relations that are most possibly faulty but do not take a huge set of candidates, we enforce fast returning from the SAT solver whenever this specific relaxation is not indeed the answer and quick finding of the faulty relations. Guided Error Localization could repair the faulty output of all sorts of errors in less than 14 seconds. 


\section{Chapter 6}

\section{Conclusions and Future Work}

We introduced the use of rich behavioral specifications extracted from method contracts for systematic data structure repair using the Alloy toolset as an enabling technology. Four repair algorithms were presented and evaluated using a text book data structure and an open-source application. Our algorithms can repair erroneous data structures with tens of nodes in seconds. The results are encouraging and show the feasibility of repairing complex structures of small sizes with a small number of faults.

The primary contributions of our repair framework, Tarmeem, are the algorithms for repair based on rich behavioral specifications and a demonstration of the feasibility and potential of such an approach, specifically for structurally complex data. The performance of our prototype is encouraging and we believe repair can increase reliability of real applications because of the following reasons:

- A deployed system that has been well-tested can be assumed to have a few errors and focusing repair on likely erroneous fields to reduce the nondeterminism in the specification is likely to provide effective pruning of the search space and to minimize the number of SAT invocations. Note 
that when no contract violation happens, no repair is performed and SAT/constraint solving is not executed. Hence the overhead of using Tarmeem with a correct program is of the same order of magnitude as that of enforcing contracts using runtime checking.

- Despite the NP hard nature of the SAT problem, advancements in SAT technology over the past decade have been substantial. In addition, our heuristics attempt to reduce the size of the SAT problem by focusing on the erroneous parts of the data structure. Our algorithms provide a solid basis for developing more effective algorithms to precisely identify the error and improve the scalability of Tarmeem.

- Our approach is not limited to repair of program state at runtime. For example, data structure repair frameworks can provide useful feedback to perform program repair and automate debugging [24].

Our current use of SAT represents a bottleneck for scaling the algorithms to larger structures. While previous work on assertion-based repair [12] suffers from ignoring post-conditions, it is approximately 10 times faster than our framework since it uses Korat [7] instead of SAT. Our limited experiments show that Tarmeem can achieve the same speed-up using Korat. In addition, we believe a tighter integration with SAT holds much promise. We are investigating techniques to guide SAT backtracking, so that SAT is not invoked multiple times to perform repair, rather the SAT search is tailored 
directly based on the repair algorithms. Moreover, we believe a use of specialized solvers, such as integer constraint solvers and string constraint solvers, in conjunction with SAT will allow us to scale contract-based repair to larger structures and to develop it into a viable technique for improving reliability of real applications. Empirical rigorous evaluation (including an evaluation on more real world applications) as well as developing better heuristics to accurately find the violating parts of the program state is a part of the future work. Using repair feedback to improve the program is another promising research avenue. 


\section{Bibliography}

[1] Alloy home page. http://alloy.mit.edu.

[2] Ext2 fsck manual page. http://e2fsprogs.sourceforge.net.

[3] Microsoft chkdsk manual page. http://support.microsoft.com/kb/ 315265.

[4] SAT4J home page. http://www.sat4j.org.

[5] Mike Barnett, K. Rustan M. Leino, and Wolfram Schulte. The Spec\# Programming System: An Overview. Construction and Analysis of Safe, Secure, and Interoperable Smart Devices, pages 49-69, 2004.

[6] Stephen M Blackburn, Robin Garner, Chris Hoffmann, Asjad M Khan, Kathryn S McKinley, Rotem Bentzur, Amer Diwan, Daniel Feinberg, Daniel Frampton, Samuel Z Guyer, Martin Hirzel, Antony Hosking, Maria Jump, Han Lee, J Eliot B Moss, Aashish Phansalkar, Darko Stefanovic, Thomas VanDrunen, Daniel von Dincklage, and Ben Wiedermann. The DaCapo Benchmarks: Java Benchmarking Development and Analysis. In Proceedings of the 21st Annual ACM SIGPLAN Conference on ObjectOriented Programming Systems, Languages, and Applications, pages 190211. ACM, 2006. 
[7] Chandrasekhar Boyapati, Sarfraz Khurshid, and Darko Marinov. Korat: Automated Testing Based on Java Predicates. In Proceedings of the International Symposium on Software Testing and Analysis (ISSTA), pages 123-133, July 2002.

[8] H. Bunke. On a Relation Between Graph Edit Distance and Maximum Common Subgraph. Pattern Recognition Letters, 18(9):689-694, 1997.

[9] Brian Demsky. Data Structure Repair Using Goal-Directed Reasoning. PhD thesis, Massachusetts Institute of Technology, January 2006.

[10] Brian Demsky and Martin Rinard. Automatic Detection and Repair of Errors in Data Structures. In Proceedings of the Conference on ObjectOriented Programming, Systems, Languages, and Applications (OOPSLA), pages $78-95,2003$.

[11] Bassem Elkarablieh. Assertion-based Repair of Complex Data Structures. PhD thesis, University of Texas at Austin, 2009.

[12] Bassem Elkarablieh, Ivan Garcia, Yuk Lai Suen, and Sarfraz Khurshid. Assertion-based Repair of Complex Data Structures. In Proceedings of the 22nd Conference on Automated Software Engineering (ASE), pages 64-73. ACM, 2007.

[13] G. Haugk, F. Lax, R. Royer, and J. Williams. The 5ESS(TM) Switching System: Maintenance Capabilities. ATEST Technical Journal, 64(6 part 2), 1985 . 
[14] Daniel Jackson. Alloy: A Lightweight Object Modeling Notation. ACM Transactions on Software Engineering and Methodology (TOSEM), 11(2), April 2002.

[15] Daniel Jackson. Software Abstractions: Logic, Language and Analysis. The MIT Press, 2006.

[16] Daniel Jackson and Alan Fekete. Lightweight Analysis of Object Interactions. In Proceedings of the 4th International Symposium on Theoretical Aspects of Computer Software, pages 492 - 513, Sendai, Japan, October 2001.

[17] Sarfraz Khurshid. Generating Structurally Complex Tests from Declarative Constraints. $\mathrm{PhD}$ thesis, Department of Electrical Engineering and Computer Science, Massachusetts Institute of Technology, December 2003.

[18] Sarfraz Khurshid, Ivan Garcia, and Yuk Lai Suen. Repairing Structurally Complex Data. In 12th SPIN Workshop on Model Checking of Software, pages 123-138, San Francisco, CA, August 2005.

[19] Sarfraz Khurshid and Darko Marinov. TestEra: Specification-based Testing of Java Programs Using SAT. Automated Software Engineering Journal, pages 403-434, 2004.

[20] Shriram Krishnamurthi, Kathi Fisler, Daniel J. Dougherty, and Daniel Yoo. Alchemy: Transmuting Base Alloy Specifications into Implemen- 
tations. In Proceedings of the 16th ACM SIGSOFT Symposium on the Foundations of Software Engineering (FSE), pages 158-169, 2008.

[21] Gary T. Leavens, Albert L. Baker, and Clyde Ruby. Preliminary Design of JML: A Behavioral Interface Specification Language for Java. Technical Report TR 98-06i, Department of Computer Science, Iowa State University, 1998.

[22] Grey T. Leavens, Albert L. Baker, and Clyde Ruby. JML: a Java Modeling Language. In Formal Underpinnings of Java Workshop, 1998.

[23] Barbara Liskov and John Guttag. Program Development in Java: Abstraction, Specification, and Object-Oriented Design. Addison-Wesley, 2000 .

[24] Muhammad Zubair Malik, Khalid Ghori, Bassem Elkarablieh, and Sarfraz Khurshid. A Case for Automated Debugging Using Data Structure Repair. In Proceedings of the 24th Conference on Automated Software Engineering (ASE), pages 620-624. IEEE, 2009.

[25] Darko Marinov and Sarfraz Khurshid. TestEra: A Novel Framework for Automated Testing of Java Programs. In Proceedings of the 16th Conference on Automated Software Engineering (ASE), pages 22-34, San Diego, CA, November 2001.

[26] Bertrand Meyer. Applying "Design by Contract". IEEE Computer, 25(10):40-51, 1992. 
[27] Samiha Mourad and Dorothy Andrews. On the Reliability of the IBM MVS/XA Operating System. IEEE Transactions on Software Engineering, 13(10), 1987.

[28] T.J. Parr and R.W. Quong. ANTLR: A Predicated-LL (k) Parser Generator. Software-Practice and Experience, 25(7):789-810, 1995.

[29] A. Sanfeliu and King-Sun Fu. Distance Measure Between Attributed Relational Graphs for Pattern Recognition. IEEE Transactions on Systems, Man, and Cybernetics, 13(3):353-362, 1983.

[30] Emina Torlak and Daniel Jackson. Kodkod: A Relational Model Finder. In Proceedings of the 13th Conference on Tools and Algorithms for Construction and Analysis of Systems (TACAS), pages 632-647, Braga, Portugal, March 2007.

[31] Jennifer Widom and Stefano Ceri. Active Database Systems: Triggers and Rules for Advanced Database Processing. Morgan Kaufmann Pub, 1996.

[32] Kuat. T. Yessenov. A Lightweight Specification Language for Bounded Program Verification. PhD thesis, MASSACHUSETTS INSTITUTE OF TECHNOLOGY, 2009.

[33] Razieh Nokhbeh Zaeem and Sarfraz Khurshid. Contract-based Data Structure Repair Using Alloy. In Proceedings of the 24th European Con- 
ference on Object-Oriented Programming (ECOOP), Maribor, Slovenia, EU, June 2010. (To appear).

[34] Razieh Nokhbeh Zaeem and Sarfraz Khurshid. Introducing SpecificationBased Data Structure Repair Using Alloy. In Proceedings of the International Conference on ASM Alloy B and Z, pages 398-399, Orford, Qubec, Canada, February 2010. 


\section{Vita}

Razieh Nokhbeh Zaeem was born in Tehran, Iran on 12 September 1984. She received the Bachelor of Science degree in Computer Engineering from Sharif University of Technology, Tehran, Iran in 2006. She was honored to bypass the graduate school admission process of the same university as a result of her outstanding performance. In 2008, she applied to the University of Texas at Austin and was admitted. She started her graduate studies in Software Engineering in September, 2008.

Permanent address: 2501 Lake Austin Blvd Apt E104

Austin, Texas 78703

This thesis was typeset with $\mathrm{H}_{\mathrm{E}} \mathrm{X}^{\dagger}$ by the author.

\footnotetext{
${ }^{\dagger} \mathrm{HT}_{\mathrm{E}} \mathrm{X}$ is a document preparation system developed by Leslie Lamport as a special version of Donald Knuth's TEX Program.
} 